\title{
Measurement of spin coherence using Raman scattering
}

\section{Journal Article}

Author(s):

Sun, Zhe; Delteil, Aymeric; Fält Stefan; Imamoglu, Atac

Publication date:

2016-06-15

Permanent link:

https://doi.org/10.3929/ethz-b-000117712

Rights / license:

In Copyright - Non-Commercial Use Permitted

Originally published in:

Physical Review B 93(24), https://doi.org/10.1103/PhysRevB.93.241302

Funding acknowledgement:

159196 - Quantum interface between solid-state spins, single photon pulses and phonons (SNF) 


\title{
Measurement of spin coherence using Raman scattering
}

\author{
Z. Sun, A. Delteil, S. Faelt, and A. Imamoğlu \\ Institute of Quantum Electronics, ETH Zurich, CH-8093 Zurich, Switzerland \\ (Received 8 April 2016; revised manuscript received 24 May 2016; published 13 June 2016)
}

\begin{abstract}
Ramsey interferometry provides a natural way to determine the coherence time of most qubit systems. Recent experiments on quantum dots, however, demonstrated that dynamical nuclear spin polarization can strongly influence the measurement process, making it difficult to extract the $T_{2}^{*}$ coherence time using standard optical Ramsey pulses. Here, we demonstrate an alternative method for spin coherence measurement that is based on first-order coherence of photons generated in spin-flip Raman scattering. We show that if a quantum emitter is driven by a weak monochromatic laser, Raman coherence is determined exclusively by spin coherence, allowing for a direct determination of spin $T_{2}^{*}$ time. When combined with coherence measurements on Rayleigh scattered photons, our technique enables us to identify coherent and incoherent contributions to resonance fluorescence, and to minimize the latter. We verify the validity of our technique by comparing our results to those determined from Ramsey interferometry for electron and heavy-hole spins.
\end{abstract}

DOI: 10.1103/PhysRevB.93.241302

A single electron or hole spin confined in a InGaAs self-assembled quantum dot (QD) is a promising candidate for the realization of quantum information processing protocols that rely on an efficient spin-photon interface [1-3]. For all of the proposed applications, understanding the nature of QD spin coherence using Ramsey and dynamical decoupling techniques is essential [4,5]. Remarkably, Ramsey interferometry implemented using optical rotation pulses in QDs is strongly influenced by dynamical nuclear spin polarization effects [6-8]. In fact, without a specifically designed pulse sequence [9], Ramsey experiments on an electron spin show a few nonsinusoidal oscillations before the signal vanishes completely on time scales that are a factor of $\sim 4$ shorter than the expected $T_{2}^{*}$ time.

In this Rapid Communication, we implement an alternative method to determine the spin coherence time of a quantum emitter that is to a large extent immune to the limitations that influence Ramsey interferometry. The principal idea behind our work is the fact that first-order coherence of spin-flip Raman scattering (inelastic scattering resulting in a change of the spin state) is determined by the coherence properties of the excitation laser field and the spin coherence $[10,11]$. Therefore, measuring the coherence time of Raman scattered photons upon excitation with a monochromatic laser field is equivalent to a measurement of the spin dephasing time. As we show below, it is essential to carry out Raman coherence measurements at a low excitation limit well below the saturation intensity in order to ensure that spin dephasing induced by Rayleigh scattering remains weak as compared to the inherent $T_{2}^{*}$ time. Moreover, dynamical nuclear spin polarization is strongly suppressed in this regime, allowing us to observe the expected Gaussian decay of the interference signal.

The experiments are based on single InGaAs QDs grown epitaxially in a $p-i-n$ structure. The QD layer is separated by a $35 \mathrm{~nm}$ tunneling barrier from the $n^{+}$back contact and $40 \mathrm{~nm}$ AlGaAs blocking barrier from the top $p^{+}$contact. The $p-i-n$ structure is placed inside a planar cavity $(Q \sim 20)$, consisting of a bottom distributed Bragg reflector (DBR) of 28 pairs and a top DBR of two pairs. A $\mathrm{ZnO}$ solid immersion lens (SIL) mounted on the top of the sample is used to increase the collection efficiency. The sample is held in a bath cryostat operating at liquid He temperature. A confocal microscope is used to excite QDs with lasers and collect scattered photons through the same objective. The reflected laser background is suppressed to about $10^{-6}$ by a cross polarization configuration [12]. The scattered photons from QDs are guided to a superconducting single-photon detector (SSPD) and recorded by a time-correlated single-photon counting module (TCSPC). A double- $\Lambda$ system for elementary optical excitations is obtained by applying an external magnetic field perpendicular to the QD growth direction (Voigt geometry). A laser that is resonant with one of the four transitions leads to resonance fluorescence (RF), including contributions from incoherent spontaneous emission, coherent Rayleigh scattering, and coherent spin-flip Raman scattering.

The experiment is first performed on a single electron charged QD in Voigt geometry. Figure 1(b) shows the relevant energy-level diagram in Voigt geometry [13]. A finite Zeeman splitting of the ground states and the excited states is generated by an external magnetic field $B_{x}$, yielding optical selection rules with four allowed transitions of identical oscillator strength. The two ground states are identified by the orientation of the electron spin, with $|\uparrow\rangle(|\downarrow\rangle)$ denoting $+1 / 2(-1 / 2)$ angular momentum projection along $B_{x}$. We set $B_{x}=4.6 \mathrm{~T}$, which gives to an electron Zeeman splitting of $22 \mathrm{GHz}$ and a hole Zeeman splitting of $8 \mathrm{GHz}$. The two vertical transitions (blue transition: $|\uparrow\rangle$ to blue trion state $\left|T_{b}\right\rangle$; red transition: $|\downarrow\rangle$ to red trion state $\left.\left|T_{r}\right\rangle\right)$ are $V$ polarized, and the two diagonal transitions (diagonal transition $1:|\downarrow\rangle$ to blue trion state $\left|T_{b}\right\rangle$; diagonal transition $2:|\uparrow\rangle$ to red trion state $\left|T_{r}\right\rangle$ ) are $H$ polarized. The pulse sequence used in the experiment is outlined in Fig. 1(c). All the laser pulses are obtained from cw lasers by electro-optical modulators with a $10^{3}$ on/off ratio. The QD is first prepared in the $|\uparrow\rangle$ state by spin pumping using a $10 \mathrm{~ns}$ laser pulse, termed the preparation pulse, tuned on resonance with the red transition. Subsequently, a $10 \mathrm{~ns}$ laser pulse resonant with the blue transition, which we refer to as the excitation laser, is applied inducing a two-color photon emission: $V(H)$-polarized emission of the center frequency $\omega_{\text {blue }}\left(\omega_{\text {diag1 }}\right)$ including coherent Rayleigh (Raman) scattering and incoherent spontaneous emission. 
(a)

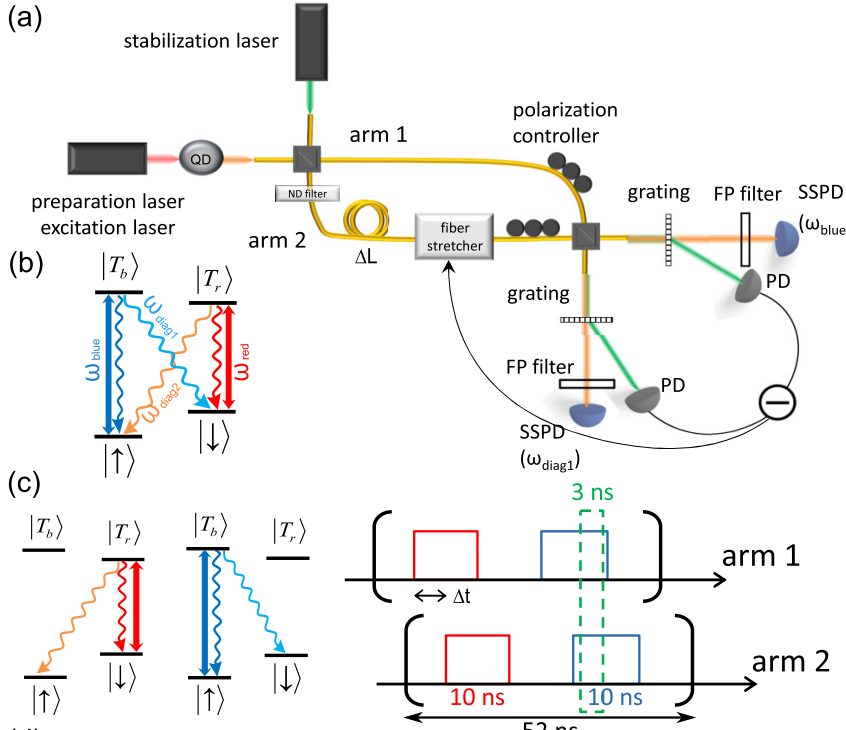

(d)
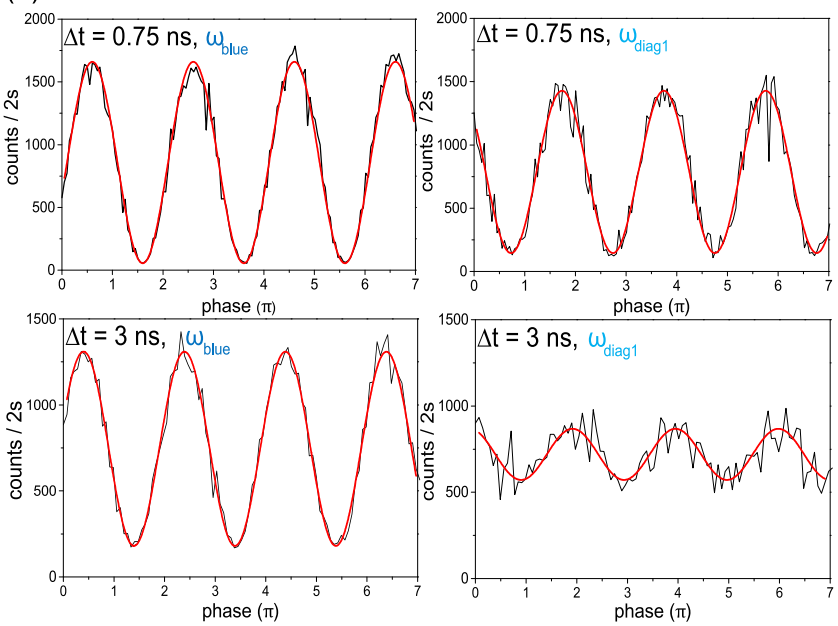

FIG. 1. (a) Sketch of the stablized Mach-Zehnder interferometer. (b) Energy-level diagram of a single electron charged QD in Voigt geometry. (c) Pulse sequence used for the first-order coherence measurement of an electron spin and relevant transitions. Red square frame: $10 \mathrm{~ns}$ preparation pulse; blue square frame: $10 \mathrm{~ns}$ excitation pulse; green dashed box: 3 ns postselected time window. The overall repetition rate is $52 \mathrm{~ns}$. (d) Count rate of SSPD as a function of phase difference, for $\Delta t=0.75 \mathrm{~ns}$ (upper row) and $\Delta t=3 \mathrm{~ns}$ (lower row) when filtering only $\omega_{\text {blue }}$ (left column) or $\omega_{\text {diag1 }}$ (right column).

As depicted in Fig. 1(a), the scattered photons are fed into one of the input ports of the stabilized Mach-Zehnder interferometer. The path length difference $\Delta L$ between the two arms leads to a time delay $\Delta t=n \Delta L / c$, where $c$ denotes the speed of light in vacuum, and $n$ denotes the refraction index of fiber. Two Fabry-Pérot filters of $1.7 \mathrm{GHz}$ linewidth select either $\omega_{\text {blue }}$ photons or $\omega_{\text {diag1 }}$ photons exclusively. A TCSPC records the photon detection events, allowing one to postselect a 3 ns overlapping time window, as shown in Fig. 1(c). We ensure that the amplitudes and polarizations of the two arms are rendered identical by introducing a variable neutral density (ND) filter in arm 2. To stabilize the path length difference, we use an active homodyne stabilization method [14] with an additional laser at a longer wavelength $\lambda_{0}$ such that it can be separated from the QD photons by a transmission grating of $1500 \ell / \mathrm{mm}$. The photodiodes (PDs) placed at the two output ports measure the intensity of the stabilization laser. A commercial electronic bias controller provides a feedback signal on a fiber stretcher placed in one arm, enabling one to lock the path length difference to an arbitrary value. Furthermore, it is possible to continuously change the path length difference by scanning the stabilization laser wavelength in a quasistatic way. A change of $d \lambda_{0}$ in the stabilization laser wavelength yields a change of $d \lambda_{0} \Delta L / \lambda_{0}$ in the path length difference of the Mach-Zehnder interferometer. As an example, Fig. 1(d) presents the count rate of SSPD in a $3 \mathrm{~ns}$ postselected time window for two particular time delays: $\Delta t=0.75 \mathrm{~ns}$ (upper row) and $\Delta t=3 \mathrm{~ns}$ (lower row), when filtering only $\omega_{\text {blue }}$ (left column) or only $\omega_{\text {diag1 }}$ (right column) photons. The visibility of $\omega_{\text {blue }}$ photons is limited by the contribution from incoherent spontaneous emission, whereas the visibility of $\omega_{\text {diag } 1}$ photons is additionally reduced due to the fact that the spin-flip Raman scattering is affected by the spin coherence.

To characterize the first-order coherence of $\omega_{\text {blue }}$ and $\omega_{\text {diag1 }}$ photons, the interference visibility is plotted as a function of the time delay $\Delta t$ for two different powers of the excitation laser corresponding to $P_{1}=0.1 P_{\text {sat }}, P_{2}=0.5 P_{\text {sat }}$, where $P_{\text {sat }}=7.2 \mathrm{nW}$ is the saturation power for the blue transition extracted from the excitation power dependent spin pumping rate $\Gamma_{\mathrm{SP}}$ as shown in Fig. 2(b) (inset). In Fig. 2(a), the visibilities are extracted from the interference fringes obtained by filtering either $\omega_{\text {blue }}$ or $\omega_{\text {diagl }}$ photons. All the data have been normalized by the interference visibility of the excitation laser in order to retain only the contribution originating from the QD scattering.

We calculate the visibility of $\omega_{\text {blue }}$ and $\omega_{\text {diag1 }}$ photons using a master equation and the quantum regression theorem (QRT) (see the Supplemental Material [15]). In the simulations, we set the lifetime of $\left|T_{b}\right\rangle$ state $T_{1}$ to $0.76 \mathrm{~ns}$ which we measured from the decay of time-resolved RF excited by a short laser pulse.

The effect of the slowly fluctuating Overhauser field is taken into account by averaging over cases with different Zeeman splittings, with a Gaussian distribution around the center value. This leads to a decay of the visibility on a time scale of $T_{2}^{*}$. The distribution of Larmor frequencies is a normal distribution with a standard deviation $\sigma=\sqrt{2} / T_{2}^{*}$. The theoretical description we use is valid in the weak excitation regime where the probability to scatter more than one photon during the relevant time scale can be neglected. This implies $\Gamma_{\mathrm{SP}} \Delta t \ll 1$.

The visibility of $\omega_{\text {blue }}\left(\omega_{\text {diag } 1}\right)$ photons can be decomposed into a coherent Rayleigh (Raman) scattering and an incoherent spontaneous emission that vanishes on the time scale of $T_{2} \leqslant$ $2 T_{1}$, where $T_{2}$ denotes the coherence time of $\left|T_{b}\right\rangle$ state. For a two-level system, the fraction of the coherent scattering is given by

$$
\frac{I^{\text {coherent }}}{I^{\text {total }}}=\frac{2 \Gamma^{2}}{2 \Gamma^{2}+\Omega^{2}}=\frac{1}{1+P / P_{\mathrm{sat}}},
$$

where $\Gamma=1 / T_{1}$ is the spontaneous emission rate of the excited state, $\Omega$ is the Rabi frequency, and $P$ is the excitation laser power [16]. Note that at low power, there is almost 

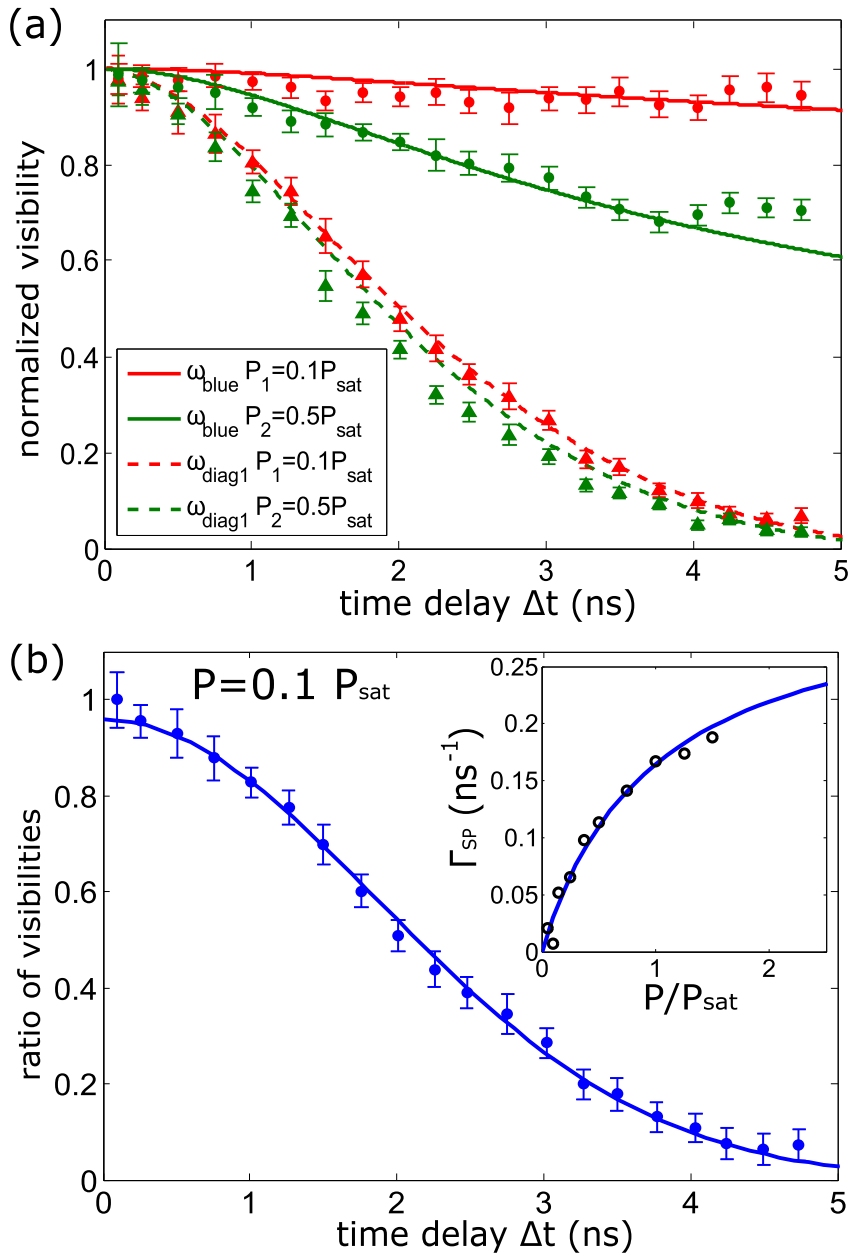

FIG. 2. (a) The normalized visibility extracted from the interference fringes of $\omega_{\text {blue }}$ photons (solid lines and circular points) and $\omega_{\text {diag1 }}$ photons (dashed lines and triangular points) as a function of the time delay $\Delta t$ for two different powers of the excitation laser. In the simulation, the fitting curve is plotted using $T_{1}=0.76 \mathrm{~ns}, T_{2}=2 T_{1}$, $T_{2}^{*}=2.4 \mathrm{~ns}$. (b) Electron spin: The ratio between the visibility of $\omega_{\text {blue }}$ photons and $\omega_{\text {diag1 }}$ photons as a function of the time delay $\Delta t$ for the excitation laser power $P=0.1 P_{\text {sat }}$. The solid curve is a Gaussian fitting of the data. Inset: Spin pumping rate as a function of the excitation laser power. The $x$ axis has been normalized by the saturation power of the blue transition $P_{\text {sat }}=7.2 \mathrm{nW}$. The fitting curve is $\Gamma_{\mathrm{SP}}=0.5 \Omega^{2} \Gamma /\left(\Gamma^{2}+2 \Omega^{2}\right)$.

no contribution from incoherent scattering for both $\omega_{\text {blue }}$ and $\omega_{\text {diag1 }}$ photons. Thus the ratio between the visibility of $\omega_{\text {diag1 }}$ photons and $\omega_{\text {blue }}$ photons reveals the spin coherence allowing for a direct extraction of $T_{2}^{*}$ by assuming Gaussian decay. Figure 2(b) is the ratio of visibilities measured with the power of the excitation laser $P=0.1 P_{\text {sat }}$. The $T_{2}^{*}$ of the electron spin extracted from a Gaussian fitting is $2.6 \mathrm{~ns}$, in agreement with previously reported values of the electron $T_{2}^{*}$ [17].

The maximum time delay $\Delta t$ at which we can still perform interference measurements is limited by the spin pumping time, preventing us from measuring decoherence times longer than a few nanoseconds. However, this limitation can be overcome by introducing a modification of the pulse sequence, which then consists of two excitation pulses of $t_{0}=3 \mathrm{~ns}$ (a)

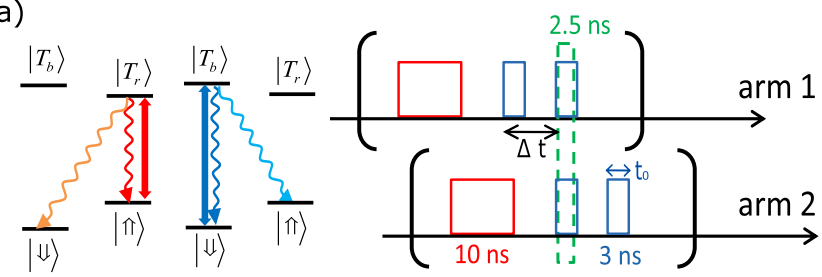

(b)

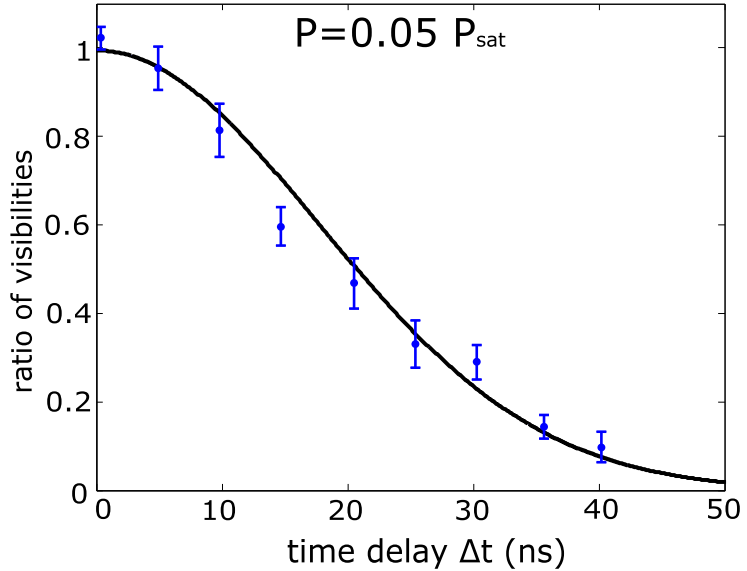

FIG. 3. (a) Pulse sequence used for the first-order coherence measurement of a hole spin and relevant transitions. Red square frame: $10 \mathrm{~ns}$ preparation pulse; blue square frames: 3 ns excitation pulse; green dashed box: $2.5 \mathrm{~ns}$ postselected time window. (b) Hole spin: The ratio between the visibility of $\omega_{\text {blue }}$ photons and $\omega_{\text {diag } 1}$ photons as a function of the time delay $\Delta t$ for the excitation laser power $P=0.05 P_{\text {sat }}$. The solid curve is a Gaussian fitting of the data.

separated by $\Delta t$, matching the delay between the two arms of the interferometer, as shown in Fig. 3(a). We then postselect the overlapping time window and display the count rate. Here, we illustrate this extension by measuring the hole spin dephasing time which is an order of magnitude longer than that of the electron. The measurement is carried out on the same QD charged with a single hole in the same magnetic field. A hole is optically injected into the QD by driving the neutral exciton resonantly. By properly choosing the gate voltage, the electron tunnels out, leaving the QD with a single excess hole whose lifetime exceeds $400 \mathrm{~ns}$ [18]. In the energy-level diagram in Fig. 3(a), $|\Uparrow\rangle$ and $|\Downarrow\rangle$ denote the hole spin states. Figure 3(b) is the ratio of visibilities measured with an excitation laser power $P=0.05 P_{\text {sat }}$. We extract $T_{2}^{*}=25.7 \mathrm{~ns}$ from a Gaussian fitting which is consistent with previously reported values of hole coherence at high magnetic fields $[8,19]$.

The spin dephasing times of both electron and hole spins determined by the first-order coherence measurements agree with what we estimated from a Ramsey experiment on the same QD (see the Supplemental Material). As mentioned earlier, the Ramsey fringes strongly deviate from a Gaussian decay of sinusoidal oscillations due to the strong hyperfine interaction. The technique presented here essentially eliminates these unwanted effects, allowing for an unambiguous extraction of $T_{2}^{*}$. This is rendered possible by using very low laser powers and, in the case of the electron spin, a pulse sequence that stays unchanged in the whole experiment in stark contrast with Ramsey interferometry which is influenced by 
delay-dependent nuclear spin polarization. Moreover, it does not need prior implementation of spin rotation and hence can be used in situations where such rotation is not applicable (e.g., to measure the quantum dot spin coherence in Faraday geometry by driving the weakly allowed diagonal transition).

Our results demonstrate that Mach-Zehnder-type singlephoton interferometry carried out on spin-flip Raman scattering can be used to measure the coherence time of a QD spin. While the use of coherent population trapping for determining spin coherence is based on the same principle
[20-22], we emphasize that the technique we present allows for a direct measurement of $T_{2}^{*}$ time that does not require the determination of the Rabi frequency associated with the driving laser field.

This work is supported by NCCR Quantum Photonics (NCCR QP), the research instrument of the Swiss National Science Foundation (SNSF), and by the Swiss NSF under Grant No. 200020-159196.

Z.S. and A.D. contributed equally to this work.
[1] W. B. Gao, P. Fallahi, E. Togan, J. Miguel-Sanchez, and A. Imamoğlu, Nature (London) 491, 426 (2012).

[2] K. De Greve et al., Nature (London) 491, 421 (2012).

[3] J. R. Schaibley, A. P. Burgers, G. A. McCracken, L.-M. Duan, P. R. Berman, D. G. Steel, A. S. Bracker, D. Gammon, and L. J. Sham, Phys. Rev. Lett. 110, 167401 (2013).

[4] N. F. Ramsey, Phys. Rev. 78, 695 (1950).

[5] K. De Greve et al., Nat. Phys. 7, 872 (2011).

[6] A. Högele, M. Kroner, C. Latta, M. Claassen, I. Carusotto, C. Bulutay, and A. Imamoğlu, Phys. Rev. Lett. 108, 197403 (2012).

[7] T. D. Ladd, D. Press, K. De Greve, P. L. McMahon, B. Friess, C. Schneider, M. Kamp, S. Höfling, A. Forchel, and Y. Yamamoto, Phys. Rev. Lett. 105, 107401 (2010).

[8] S. G. Carter, S. E. Economou, A. Greilich, E. Barnes, T. Sweeney, A. S. Bracker, and D. Gammon, Phys. Rev. B 89, 075316 (2014).

[9] R. Stockill, C. L. Gall, C. Matthiesen, L. Huthmacher, E. Clarke, M. Hugues, and M. Atatüre, arXiv:1512.01811.

[10] G. Fernandez, T. Volz, R. Desbuquois, A. Badolato, and A. Imamoğlu, Phys. Rev. Lett. 103, 087406 (2009).

[11] T. M. Sweeney et al., Nat. Photonics 8, 442 (2014).

[12] A. N. Vamivakas, Y. Zhao, C.-Y. Lu, and M. Atatüre, Nat. Phys. 5, 198 (2009).
[13] X. Xu, Y. Wu, B. Sun, Q. Huang, J. Cheng, D. G. Steel, A. S. Bracker, D. Gammon, C. Emary, and L. J. Sham, Phys. Rev. Lett. 99, 097401 (2007).

[14] D. Pulford, C. Robillard, and E. Huntington, Rev. Sci. Instrum. 76, 063114 (2005).

[15] See Supplemental Material at http://link.aps.org/supplemental/ 10.1103/PhysRevB.93.241302 for theoretical calculation of the interference visibility and experimental data obtained with standard and modified Ramsey interferometry.

[16] R. Loudon, The Quantum Theory of Light (Oxford University Press, Oxford, U.K., 2000).

[17] A. Bechtold, D. Rauch, F.-X. Li, T. Simmet, P.-L. Ardelt, A. Regler, K. Müller, N. A. Sinitsyn, and J. J. Finley, Nat. Phys. 11, 1005 (2015).

[18] A. Delteil, Z. Sun, W. B. Gao, E. Togan, S. Faelt, and A Imamoğlu, Nat. Phys. 12, 218 (2015).

[19] A. Greilich, S. G. Carter, D. Kim, A. S. Bracker, and D. Gammon, Nat. Photonics 5, 702 (2011).

[20] A. Imamoğlu, Phys. Status Solidi B 243, 3725 (2006).

[21] D. Brunner, B. D. Gerardot, P. A. Dalgarno, G. Wüst, K. Karrai, N. G. Stoltz, P. M. Petroff, and R. J. Warburton, Science 325, 70 (2009).

[22] K. M. Weiss, J. M. Elzerman, Y. L. Delley, J. Miguel-Sanchez, and A. Imamoğlu, Phys. Rev. Lett. 109, 107401 (2012). 